http://jmscr.igmpublication.org/home/

ISSN (e)-2347-176x ISSN (p) 2455-0450

crossref DOI: https://dx.doi.org/10.18535/jmscr/v8i3.08

Journal Of Medical Science And Clinical Research

\title{
To Study the Correlation of Serum Adiponectin with Visceral Body Fat and Carotid Intima Medial Thickness in Non Diabetic Patients (30-60 Years)
}

\author{
Authors \\ Dr Jyoti Verma ${ }^{1}$, Dr Arun Gogna ${ }^{2}$ \\ ${ }^{1,2}$ Department of Medicine, VMMC AND SJH
}

\begin{abstract}
Introduction: Cardiovascular disease is the leading cause of disability adjusted life years in developed and developing countries. Traditional cardiovascular risk factors such as obesity, hypertension and disturbances of glucose and lipid metabolism are commonly clustered and insulin resistance has been considered a relevant pathophysiological link. However, a number of cardiovascular events occur in absence of these risk factors indicating that there might be a substantial need for identification of these novel factors.

Adipose tissue secretes adiponectin which participates in energy homeostasis and enhances insulin sensitivity. An inverse association of adiponectinemia with obesity, insulin resistance, inflammation, type 2 diabetes and atherosclerosis has been reported. Adiponectin has been associated with carotid intima medial thickness(CIMT), mainly in high cardiovascular risk population

Therefore, we performed this study to assess the association of adiponectin with CIMT in Indian subjects without diabetes or cardiovascular disease

Material and Method: We conducted a cross sectional observational study at Safdarjung hospital.

Results: Our study proved that adiponectin can be used as marker of subclinical atherosclerosis in non diabetic patients. There is an inverse correlation of serum adiponectin with CIMT ( $p<0.001)$. However, we did not observe any correlation between serum adiponectin with visceral body fat and BMI.

Summary: Hence, we can say that as the level of serum adiponectin decreases CIMT increases and vice versa. Therefore serum adiponectin can be used as the marker of subclinical atherosclerosis.
\end{abstract}

\section{Introduction}

Cardiovascular disease is the leading cause of disability adjusted life years in developed and developing countries. ${ }^{1}$ Traditional cardiovascular risk factors such as obesity, hypertension and disturbances of glucose and lipid metabolism are commonly clustered and insulin resistance has been considered a relevant pathophysiological link. However, a number of cardiovascular events occur in absence of these risk factors ${ }^{2}$ indicating that there might be a substantial need for identification of these novel factors.

Inflammatory adipose tissue derived cytokines detected in circulation have been proposed as novel biomarkers of cardiometabolic risk. ${ }^{3}$ These biomarkers are increased in obesity and may play a role in atherogenesis. Adiponectin participates in energy homeostasis and enhances insulin sensitivity. An inverse association of adiponectinemia with obesity, insulin resistance, inflammation, type 2 diabetes and atherosclerosis has been reported. ${ }^{4-6}$ Adiponectin has been associated with CIMT, mainly in high cardiovascular risk population. However, whether adiponectin concentration could be potential early marker of atherogenesis in low to moderate cardiovascular risk population is open to question. Carotid intimal media thickness has been considered as a non-invasive surrogate measurement of subclinical atherosclerosis. 
Although there is no consensual definition regarding a cut off for CIMT. Many study found that demographic and traditional cardiovascular risk factors did not explain the majority of common carotid intimal media thickness, which also reinforces the need to study novel risk factors. ${ }^{8}$ An objective of ELSA-Brasil is to examine surrogates of cardiovascular disease and novel biomarkers of atherosclerosis. ${ }^{9}$ The ELSABrasil study group has already reported normal ranges for CIMT according to sex, age and race in large Brazilian samples and an association of CIMT with body adiposity was found. ${ }^{10,8}$ in this Brazilian analysis, they hypothesized that independent of adiposity, the adiponectin concentration could help to identify subclinical atherosclerosis in ELSA-Brasil participants.

Adiponectin has been associated with CIMT, mainly in high cardiovascular risk population. However, whether adiponectin concentration could be potential early marker of atherogenesis in low to moderate cardiovascular risk population is open to question. There is paucity of Indian studies correlating serum adiponectin with visceral body fat and carotid intimal media thickness .Therefore, we intend to assess the association of adiponectin with CIMT in individuals without diabetes or cardiovascular disease and we intend to study the correlation of serum adiponectin with visceral body fat and carotid intimal media thickness in non-diabetic Indian population.

\section{Materials and Methods}

Set Up: Study will be conducted at VMMC and Safdarjang Hospital at medicine department

Study Subjects: 65 patients

Formula used is

$$
\begin{aligned}
& N=\left(\frac{z_{\mathrm{a}}+z_{\rho}}{C(r)}\right)^{2}+3 \\
& C(r)=\frac{1}{2} \log _{\frac{1}{1}} \frac{1+r}{1-r}
\end{aligned}
$$

Study Design:Cross sectional observational study Inclusion Criteria: Age Group -30-60 year
Non diabetic patients

Exclusion Criteria: Following patients will be excluded from study:

○ Patients on steroids

- Patients on NSAIDS

- Patients with chronic kidney disease

- Patients with chronic heart failure

- Patients with hypothyroidism

- Patients with Cushing disease

- Patients with coronary artery disease

- Smokers

○ Patients with past history of cerebrovascular accidents

\section{Methodology}

All issues including ethical issues of the thesis have been evaluated by the Institutional Review Board and have been approved. Written informed consent was obtained for each case. Patients were recruited after applying the inclusion and exclusion criteria and subjected to detailed history specially concentrating on age and gender

Serum Adiponectin: measured by ELISA method using commercially available kits.

\section{Sample Collection and Storage}

Venous blood samples were collected at the time of admission using full aseptic measures, centrifuged at $3000 \mathrm{rpm}$ for 5 minutes. Supernatant was separated as quickly as possible and then stored in vertical plain vials at -20 degree $\mathrm{C}$ till processing was done.

\section{Radiological Investigation}

\section{Carotid Intimal Medial Thickness}

The patientwere made to lie supine on the examination couch with their head turned 45 degree away from the side being scanned. The sonographers used to obtain longitudinal B mode images of left and right common carotid arteries, immediately proximal to carotid bifurcation. Sonographers used to adjust focus and gain settings to optimize far wall echoes and to minimize noise in the arterial lumen. ${ }^{8}$

Sonographic index for abdominal fat indices-

Visceral Fat: Visceral fat thickness is defined as distance between lumbar spine and posterior 
surface of rectus abdominis muscle. This was measured with $3.5 \mathrm{MHz}$ or $3.75 \mathrm{MHz}$ probe $1.5 \mathrm{~cm}$ above umbilicus at xiphoumbilical line or midway between xiphoid process and umbilicus. A cut off value of $6.9 \mathrm{~cm}$ was proposed to discriminate significant visceral body adiposity.

Body Mass Index was calculated by weight in $\mathrm{kg}$ divided by square of height in metre. Body weight and height were measured using calibrated electronic scales and a fixed rigid stadiometer, while individuals wore light clothing without shoes.

Institutional Ethics Committee Clearance: Approval will be sought from the institutional Ethics Committee before the start of the study

\section{Results}

Analysis of observation and data is as follows:

Distribution of patient into quartiles depending on serum adiponectin and CIMT

Table 1 Age Characteristics of the study population in various Quartiles depending on serum Adiponectin Values

\begin{tabular}{|c|c|c|c|c|c|}
\hline & \multicolumn{4}{|c|}{ S.ADIPONECTIN(microgm/ml) } & \\
\hline AGE (years) & $\begin{array}{c}\text { QUARTILE } 1 \\
(<=1.015)\end{array}$ & $\begin{array}{c}\text { Quartile } 2 \\
(1.016-3)\end{array}$ & $\begin{array}{l}\text { Quartile } 3 \\
(3.1-5.55)\end{array}$ & $\begin{array}{c}\text { Quartile } 4 \\
(>5.55)\end{array}$ & $P$ value \\
\hline & & & & & .905 \\
\hline SAMPLE SIZE & 17 & 16 & 16 & 16 & \\
\hline $\mathrm{MEAN} \pm \mathrm{SD}$ & $45.71 \pm 10.72$ & $42.94 \pm 9.46$ & $43.62 \pm 8.65$ & $44.06 \pm 8.93$ & \\
\hline MEDIAN & 47 & 41 & 42 & 43 & \\
\hline MIN- MAX & $30-60$ & $30-60$ & $31-60$ & $32-59$ & \\
\hline INTERQUARTILE RANGE & $35.750-55.250$ & $36-50$ & $37-50.500$ & $37-50$ & \\
\hline
\end{tabular}

Table 2 Age Characteristics of the study population in various quartiles Depending On CIMT

\begin{tabular}{|c|c|c|c|c|c|}
\hline & \multicolumn{4}{|c|}{ S.ADIPONECTIN(microgm/ml) } & \multirow[b]{2}{*}{$P$ value } \\
\hline AGE (years) & $\begin{array}{c}\text { QUARTILE } 1 \\
(<=.056)\end{array}$ & $\begin{array}{l}\text { Quartile } 2 \\
(.057-.07) \\
\end{array}$ & $\begin{array}{l}\text { Quartile } 3 \\
(.071-.08) \\
\end{array}$ & $\begin{array}{c}\text { Quartile } 4 \\
(>.08)\end{array}$ & \\
\hline & & & & & \multirow[t]{6}{*}{.646} \\
\hline SAMPLE SIZE & 17 & 18 & 16 & 14 & \\
\hline MEAN \pm SD & $46 \pm 8.85$ & $43.28 \pm 9.34$ & $44.62 \pm 9.17$ & $42.29 \pm 10.55$ & \\
\hline MEDIAN & 46 & 38.5 & 41 & 38.5 & \\
\hline MIN- MAX & $30-60$ & $30-58$ & $32-60$ & $30-60$ & \\
\hline INTERQUARTILE RANGE & $40.250-51$ & $36-51$ & $38.500-52.500$ & $32-51$ & \\
\hline
\end{tabular}

Table 3. Age Distribution (Year)* Adiponectin (microgram/ml)

\begin{tabular}{|c|c|c|c|c|c|c|c|}
\hline & \multicolumn{4}{|c|}{ S.ADIPONECTIN(microgm/ml) } & \multirow[t]{2}{*}{ Total } & \multirow[t]{2}{*}{$\mathrm{P}$ value } \\
\hline & & $\begin{array}{l}\text { Quartile 1 } \\
(<=1.015)\end{array}$ & $\begin{array}{l}\text { Quartile2 } \\
(1.016-3)\end{array}$ & $\begin{array}{l}\text { Quartile 3 } \\
(3.1-5.55)\end{array}$ & $\begin{array}{l}\text { Quartile } 4 \\
(>5.55)\end{array}$ & & \\
\hline \multirow{3}{*}{$\begin{array}{l}\text { AGE } \\
\text { DISTRIBUTION }\end{array}$} & $30-40$ & $7(41.18 \%)$ & $8(50.00 \%)$ & $8(50.00 \%)$ & $7(43.75 \%)$ & $30(46.15 \%)$ & \multirow[t]{4}{*}{0.790} \\
\hline & $41-50$ & $3(17.65 \%)$ & $4(25.00 \%)$ & $4(25.00 \%)$ & $6(37.50 \%)$ & $17(26.15 \%)$ & \\
\hline & $51-60$ & $7(41.18 \%)$ & $4(25.00 \%)$ & $4(25.00 \%)$ & $3(18.75 \%)$ & $18(27.69 \%)$ & \\
\hline \multicolumn{2}{|l|}{ TOTAL } & $17(100.00 \%)$ & $16(100.00 \%)$ & $16(100.00 \%)$ & $16(100.00 \%)$ & $65(100.00 \%)$ & \\
\hline
\end{tabular}

Table 4. Age Distribution (years) * CIMT

\begin{tabular}{|l|c|c|c|c|c|c|c|}
\hline \multicolumn{2}{|c|}{} & \multicolumn{3}{c|}{ CIMT(cm) } & \multirow{2}{*}{ TOTAL } & \multirow{2}{*}{ P value } \\
\cline { 3 - 7 } \multicolumn{2}{c|}{} & Quartile 1 (<=.056) & $\begin{array}{c}\text { Quartile 2 } \\
(.057-.07)\end{array}$ & $\begin{array}{c}\text { Quartile 3 } \\
(.071-.08)\end{array}$ & $\begin{array}{c}\text { Quartile } 4 \\
(>.08)\end{array}$ & \\
\hline \multirow{2}{*}{$\begin{array}{l}\text { AGE } \\
\text { DISTRIBUTION }\end{array}$} & $30-40$ & $4(23.53 \%)$ & $10(55.56 \%)$ & $8(50.00 \%)$ & $8(57.14 \%)$ & $30(46.15 \%)$ & 0.111 \\
\cline { 2 - 7 } & $41-50$ & $9(52.94 \%)$ & $3(16.67 \%)$ & $4(25.00 \%)$ & $1(7.14 \%)$ & $17(26.15 \%)$ \\
\cline { 2 - 7 } & $51-60$ & $4(23.53 \%)$ & $5(27.78 \%)$ & $4(25.00 \%)$ & $5(35.71 \%)$ & $18(27.69 \%)$ \\
\hline \multicolumn{2}{|l|}{ TOTAL } & $17(100.00 \%)$ & $18(100.00 \%)$ & $16(100.00 \%)$ & $14(100.00 \%)$ & $65(100.00 \%)$ & \\
\hline
\end{tabular}


Table 5. Distribution of CIMT in Various Quartiles Depending on Adiponectin Values

\begin{tabular}{|l|c|c|c|c|c|}
\hline & \multicolumn{4}{|c|}{ S.ADIPONECTIN(microgm/ml) } & Quartile 3 \\
\cline { 1 - 4 } & $\begin{array}{c}\text { Quartile 1 } \\
(<=1.015)\end{array}$ & $\begin{array}{c}\text { Quartile 2 } \\
(1.016-3)\end{array}$ & $\begin{array}{c}\text { Quartile 4 } \\
(>5.55)\end{array}$ & P value \\
\hline SAMPLE SIZE & 17 & 16 & 16 & 16 & 0.0003 \\
\hline MEAN \pm SD & $0.08 \pm 0.02$ & $0.07 \pm 0.02$ & $0.07 \pm 0.02$ & $0.06 \pm 0.01$ & \\
\hline MEDIAN & 0.08 & 0.08 & 0.06 & 0.05 & \\
\hline MIN-MAX & $0.04-0.12$ & $0.04-0.12$ & $0.04-0.12$ & $0.04-0.08$ & \\
\hline INTERQUARTILE RANGE & $0.0780-0.0903$ & $0.0580-0.0800$ & $0.0600-0.0800$ & $0.0450-0.0700$ & \\
\hline
\end{tabular}

Table 6. Distribution of Adiponectin in various quartiles depending on CIMT Values

\begin{tabular}{|l|c|c|c|c|c|}
\hline & \multicolumn{4}{|c|}{ CIMT (cm) } & p value \\
\hline $\begin{array}{l}\text { S.ADIPONECTIN } \\
(\text { microgm/ml) }\end{array}$ & $\begin{array}{c}\text { Quartile 1 } \\
(<=.056)\end{array}$ & $\begin{array}{c}\text { Quartile 2 } \\
(.057-.07)\end{array}$ & $\begin{array}{c}\text { Quartile 3 } \\
(.071-.08)\end{array}$ & $\begin{array}{c}\text { Quartile 4 } \\
(>.08)\end{array}$ & $<.0001$ \\
\hline SAMPLE SIZE & 17 & 18 & 16 & 14 \\
\hline MEAN \pm SD & $15.87 \pm 16.6$ & $4.23 \pm 2.52$ & $3.76 \pm 4.78$ & $1.16 \pm 0.92$ \\
\hline MEDIAN & 9.5 & 4.72 & 2.59 & 0.87 \\
\hline MIN-MAX & $0.96-58.5$ & $0.84-9$ & $0.2-19.4$ & $0.25-3.39$ \\
\hline INTERQUARTILE RANGE & $2.175-26.550$ & $1.505-5.800$ & $1.052-3.858$ & $0.640-1.655$ & \\
\hline
\end{tabular}

Table 7 Sex * Adiponectin

\begin{tabular}{|l|l|c|c|c|c|c|c|}
\hline \multirow{2}{*}{} & \multicolumn{5}{|c|}{ S.ADIPONECTIN(microgm/ml) } & \multirow{2}{*}{ Total } & P value \\
\cline { 3 - 7 } \multicolumn{2}{|c|}{} & $\begin{array}{c}\text { 1) Quartile 1 } \\
(<=1.015)\end{array}$ & $\begin{array}{c}\text { 2) Quartile } \\
2(1.016-3)\end{array}$ & $\begin{array}{c}\text { 3) Quartile } \\
3(3.1-5.55)\end{array}$ & $\begin{array}{c}\text { 4) Quartile } \\
4(>5.55)\end{array}$ & & \\
\hline SE & F & $7(41.18 \%)$ & $5(31.25 \%)$ & $4(25.00 \%)$ & $3(18.75 \%)$ & $19(29.23 \%)$ & 0.533 \\
\cline { 2 - 7 } X & M & $10(58.82 \%)$ & $11(68.75 \%)$ & $12(75.00 \%)$ & $13(81.25 \%)$ & $46(70.77 \%)$ & \\
\hline \multicolumn{2}{|l|}{ TOTAL } & $17(100.00 \%)$ & $16(100.00 \%)$ & $16(100.00 \%)$ & $16(100.00 \%)$ & $65(100.00 \%)$ & \\
\hline
\end{tabular}

Table 8 Sex $*$ CIMT

\begin{tabular}{|c|c|c|c|c|c|c|c|}
\hline & & \multicolumn{4}{|c|}{$\mathrm{CIM}(\mathrm{cm})$} & \multirow[b]{2}{*}{ Total } & \multirow[b]{2}{*}{$P$ value } \\
\hline & & $\begin{array}{l}\text { 1) Quartile } 1 \\
(<=.056)\end{array}$ & $\begin{array}{c}\text { 2) Quartile 2( } \\
.057-.07)\end{array}$ & $\begin{array}{l}\text { 3) Quartile } \\
\text { 3(.071-.08) }\end{array}$ & $\begin{array}{l}\text { 4) Quartile } \\
4(>.08)\end{array}$ & & \\
\hline \multirow[t]{2}{*}{ SEX } & $\mathrm{F}$ & $3(17.65 \%)$ & $5(27.78 \%)$ & $4(25.00 \%)$ & $7(50.00 \%)$ & $19(29.23 \%)$ & \multirow{3}{*}{0.243} \\
\hline & $\mathrm{M}$ & $14(82.35 \%)$ & $13(72.22 \%)$ & $12(75.00 \%)$ & $7(50.00 \%)$ & $46(70.77 \%)$ & \\
\hline \multicolumn{2}{|c|}{ TOTAL } & $17(100.00 \%)$ & $18(100.00 \%)$ & $16(100.00 \%)$ & $14(100.00 \%)$ & $65(100.00 \%)$ & \\
\hline
\end{tabular}

Table 9 Correlation between Serum Adiponectin and CIMT

\begin{tabular}{|l|c|c|}
\hline Correlation between S adiponectin and CIMT & & \\
\hline Spearman's coefficient of rank correlation (rho) & P value & $95 \%$ Confidence Interval for rho \\
\hline-0.569 & $<0.0001$ & -0.714 to -0.378 \\
\hline
\end{tabular}

Table 10. Visceral Body Fat $(\mathrm{cm}) *$ Serum Adiponectin

\begin{tabular}{|c|c|c|c|c|c|}
\hline & \multicolumn{4}{|c|}{ S.ADIPONECTIN(microgm/ml) } & \\
\hline VISCREAL BODY FAT & $\begin{array}{l}\text { Quartile 1 } \\
(<=1.015)\end{array}$ & $\begin{array}{c}\text { Quartile 2 } \\
(1.016-3)\end{array}$ & $\begin{array}{l}\text { Quartile 3 } \\
(3.1-5.55)\end{array}$ & $\begin{array}{l}\text { Quartile } 4 \\
(>5.55)\end{array}$ & $P$ value \\
\hline SAMPLE SIZE & 17 & 16 & 16 & 16 & \multirow{5}{*}{0.231} \\
\hline $\mathrm{MEAN} \pm \mathrm{SD}$ & $5.5 \pm 0.72$ & $5.77 \pm 0.57$ & $5.68 \pm 0.8$ & $6.08 \pm 1.06$ & \\
\hline MEDIAN & 5.5 & 5.8 & 5.6 & 5.9 & \\
\hline MIN-MAX & $4.07-6.8$ & $4.9-7.2$ & $4.6-7.22$ & $4.8-7.9$ & \\
\hline INTERQUARTILE RANGE & $4.900-5.975$ & $5.335-6.050$ & $5.050-6.150$ & $5.115-7$ & \\
\hline
\end{tabular}


Table 11 BMI $\left(\mathrm{kg} / \mathrm{m}^{2}\right) *$ Serum Adiponectin

\begin{tabular}{|c|c|c|c|c|c|}
\hline & \multicolumn{4}{|c|}{ S.ADIPONECTIN (microgm/ml) } & \\
\hline & $\begin{array}{l}\text { Quartile 1 } \\
(<=1.015)\end{array}$ & $\begin{array}{c}\text { Quartile } 2 \\
(1.016-3) \\
\end{array}$ & $\begin{array}{l}\text { Quartile 3 } \\
(3.1-5.55) \\
\end{array}$ & $\begin{array}{c}\text { Quartile } 4 \\
(>5.55)\end{array}$ & $P$ value \\
\hline BMI $(\mathrm{kg} / \mathrm{m} 2)$ & & & & & 0.58 \\
\hline SAMPLE SIZE & 17 & 16 & 16 & 16 & \\
\hline MEAN \pm SD & $21.72 \pm 1.54$ & $21.96 \pm 1.58$ & $22.34 \pm 1.53$ & $22.39 \pm 1.73$ & \\
\hline MEDIAN & 21.5 & 21.6 & 22 & 22.4 & \\
\hline MIN-MAX & $19.6-25$ & $20.5-27.3$ & $19.5-26$ & $19.1-25.5$ & \\
\hline INTERQUARTILE RANGE & $20.725-22.625$ & $21.150-22.200$ & $21.500-23$ & $21.300-23.550$ & \\
\hline
\end{tabular}

Table 12 Correlation by BMI and Serum Adiponectin

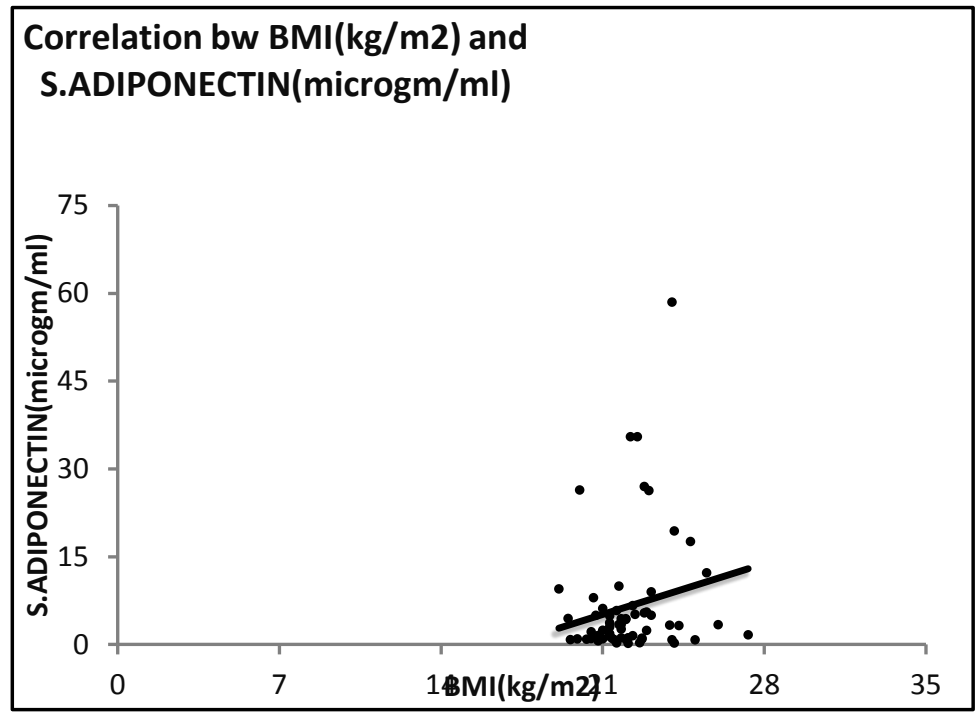

\section{Conclusion}

Following conclusions can be drawn from our study:

Adiponectin may be useful as a marker of subclinical atherosclerosis in individuals with low or moderate cardiovascular risk.

In our study we found that as the level of serum APN was decreasing, CIMT was increasing and vice versa.

There was significant inverse association of serum APN with CIMT with $p$ value of $<0.0001$

The age and gender of the population had no significant impact on the values of serum APN and CIMT. However we did not observe any correlation between serum APN with visceral body fat and BMI.

Further prospective studies are needed to confirm this association of serum APN with CIMT and the role that it plays in subclinical atherosclerosis and cardiovascular diseases.
In conclusion we can say that as the level of serum adiponectin decreases, CIMT increases and vice versa. Because of this association serum adiponectin can be used as a marker of subclinical atherosclerosis

\section{Acknowledgement}

I extend a very sincere thank to THE GREAT ALMIGHTY, and then to my guide, my parents, and my colleagues who supported me and encouraged me to do this research work.

\section{Authors' Contributions}

"Author A (Dr. JYOTI VERMA) designed the study, performed the statistical analysis, wrote the protocol, and wrote the first draft of the manuscript. Author B (Dr. ARUN GOGNA) managed the analyses of the study. All authors read and approved the final manuscript." 


\section{Consent}

All authors declare that 'written informed consent was obtained from the patient (or other approved parties) for publication of this case report and accompanying images. A copy of the written consent is available for review by the Editorial office/Chief Editor/Editorial Board members of this journal.

\section{Ethical Approval}

All authors hereby declare that all experiments have been examined and approved by the appropriate ethics committee and have therefore been performed in accordance with the ethical standards laid down in the 1964 Declaration of Helsinki.

\section{References}

1. Murray CJ, Barber RM, Foreman KJ, Abbasoglu Ozgoren A, Abd-Allah F, Abera $\mathrm{SF}$, et al. Global, regional, and national disability adjusted life years (DALYs) for 306 diseases and injuries and healthy life expectancy (HALE) for 188 countries, 1990-2013: quantifying the epidemiological transition. Lancet. Epub ahead of print 26 August 2015. DOI:10.1016/S0140-6736(15) 1340-X

2. Khot UN, Khot MB, Bajzer CT, Sapp SK, Ohman EM, Brener SJ,. et al. Prevalance of conventional risk factors in patients with coronary heart disease. JAMA 2003; 290:898-904

3. Hlatky MA, Greenland P, Arnett DK, Ballantyne CM, Criqui MH, Elkind MS et al. Criteria for evaluation of novel markers of cardiovascular risk: A scientific statement from the American Heart Association. Circulation 2009,119: 2048-2416

4. Lindberg S, Jensen JS, Bjerre M, Pedersen SH, Frystyk J, Flyvbjerg A et al. Adiponectin, type 2 diabetes and cardiovascular risk. Ear J Prev Cardiol 2015; 22:276-283

5. Maahs DM, Ogden LG, Kinney GL, et al. Low plasma adiponectin levels predict progression of coronary artery calcification. Circulation 2005;111: 747-753

6. Dullart RPF, Vries R, Tol A and Wim J Sluiter. Lower plasma adiponectin is a marker of increase intima media thickness associated with type 2 Diabetes mellitus and with male gender. Eur J Endocrinol 2007; 156: 387-394

7. Stein JH, Korcarz CE, Hurst RT, Lonn E, Kendall CB, Mohler ER, et al. use of carotid ultrasound to identify subclinical vascular disease and evaluate cardiovascular disease risk: A consensus Statement from the American Society of Echocardiography Carotid Intima Media Thickness Task Force endorsed by the society for Vascular Medicine. J Am Soc Echocardiogr 2007;21:93-111.

8. Santos IS, Alencar AP, Rundek T et al. Low impact of traditional risk factors on carotid intima medial thickness: The ELSA-Brasil cohort. Arterioselerthromb Vasc Biol 2015; 35:2054-2059.

9. Bianca de Almeida-Pititto, Fernando Flexa Ribeiro-Filho, Marcio Sommer Bittencourt, Paulo A. Lotufo, Isabela Bensenor, and Sandra R. G. Novel markers of cardiometabolic risk are associated with plasma glucose within non diabetic range. The Brazilian Longitudinal Study of Adult Health- ELSA-Brasil. Diabetes Res Clin Pract 2015; 109: 110-116.

10. Santos IS, Bittencourt MS, Oliveira IR, Souza AG, Meireles DP, Rundek T et al. Carotid intima medial thickness value distribution in the Brazilian Longitudinal Study of Adult Health (ELSA-Brasil): Atherosclerosis 2014;237:227-235. 